\title{
Correction to: On Stacked Planar Central Configurations with Five Bodies when One Body is Removed
}

\author{
Antonio Carlos Fernandes ${ }^{1}$ • Luis Fernando Mello ${ }^{1}$
}

\section{Correction to: Qual. Theory Dyn. Syst. (2013) 12:293-303 https://doi.org/10.1007/s12346-012-0084-y}

The final part of the proof of Lemma 2.7 in [3] is not correct as pointed out in [1]. The correct part, after Eq. (12), is the following one.

Suppose that $r_{5} \neq(0,0)$. Then, from Eq. (12), we have $d_{6}^{3} \lambda+M=0$, which is equivalent to

$$
\frac{\lambda}{M}+R=0
$$

where $R=d_{6}^{-3}$, for every mass $m_{5}$. The last equation can be written as

$$
R-\frac{\sum_{1 \leq i<j \leq 5} \frac{m_{i} m_{j}}{r_{i j}}}{\sum_{1 \leq i<j \leq 5} m_{i} m_{j} r_{i j}^{2}}=0
$$

which is equivalent to

$$
R \sum_{1 \leq i<j \leq 5} m_{i} m_{j} r_{i j}^{2}-\sum_{1 \leq i<j \leq 5} \frac{m_{i} m_{j}}{r_{i j}}=0 .
$$

The original article can be found online at https://doi.org/10.1007/s12346-012-0084-y.

Luis Fernando Mello

1fmelo@unifei.edu.br

Antonio Carlos Fernandes

acfernandes@unifei.edu.br

1 Instituto de Matemática e Computação, Universidade Federal de Itajubá, Avenida BPS 1303,

Pinheirinho, CEP 37.500-903 Itajubá, MG, Brazil 
Separating $m_{5}$ in the last equation, we have

$$
m_{5}\left(R \sum_{i=1}^{4} m_{i} d_{6}^{2}-\sum_{i=1}^{4} \frac{m_{i}}{d_{6}}\right)+R \sum_{1 \leq i<j \leq 4} m_{i} m_{j} r_{i j}^{2}-\sum_{1 \leq i<j \leq 4} \frac{m_{i} m_{j}}{r_{i j}}=0 .
$$

In the last equation, the factor multiplying $m_{5}$ is null, then we get

$$
R \sum_{1 \leq i<j \leq 4} m_{i} m_{j} r_{i j}^{2}-\sum_{1 \leq i<j \leq 4} \frac{m_{i} m_{j}}{r_{i j}}=0,
$$

which is equivalent to

$$
R-\frac{\sum_{1 \leq i<j \leq 4} \frac{m_{i} m_{j}}{r_{i j}}}{\sum_{1 \leq i<j \leq 4} m_{i} m_{j} r_{i j}^{2}}=0
$$

Note that the quotient in the last equation is the Lagrange multiplier of the four cocircular bodies divided by $\mathcal{M}=m_{1}+m_{2}+m_{3}+m_{4}$. Let $\lambda_{4}$ be this Lagrange multiplier. So the last equation can be written as

$$
R+\frac{\lambda_{4}}{\mathcal{M}}=0
$$

Since the four co-circular bodies are in a convex central configuration with positions disposed counterclockwise, the following inequalities must hold (see for instance [2] p. 349)

$$
R_{13}, R_{24}<-\frac{\lambda_{4}}{\mathcal{M}}<R_{12}, R_{14}, R_{23}, R_{34}
$$

On the other hand, using the perpendicular bisector theorem, we see that in a cocircular central configuration the center of the circle belongs to the interior of the convex hull of the quadrilateral, see [2]. Thus, from the geometry of a quadrilateral inscribed in the circle of radius $d_{6}$ with the center of the circle inside the convex hull of the quadrilateral, at least one side is greater than $\sqrt{2} d_{6}$. Thus, Eq. (13) is never satisfied, because the four co-circular bodies are in a convex central configuration. Therefore, in order to satisfy (12) we must have $r_{5}=(0,0)=\mathcal{C}$ and the proof is complete.

\section{References}

1. Chen, K.C., Hsiao, J.S.: Strictly convex central configurations of the planar five-body problem. Trans. Am. Math. Soc. 370, 1907-1924 (2018)

2. Cors, J.M., Roberts, G.: Four-body co-circular central configurations. Nonlinearity 25, 343-370 (2012)

3. Fernandes, A.C., Mello, L.F.: On stacked planar central configurations with five bodies when one body is removed. Qual. Theory Dyn. Syst. 12, 293-303 (2013) 